\title{
A Comparison Between Grounded and Floating Shield Inductors for $\mathrm{mmW}$ VCOs
}

\author{
José Luis González, Xavier Aragonés, Marc Molina \\ Electronic Engineering Department \\ Universitat Politècnica de Catalunya \\ Barcelona, Spain
}

\author{
Baudouin Martineau, Didier Belot \\ ST Microelectronics \\ Crolles, France
}

\begin{abstract}
A floating-shield inductor implemented in CMOS process is compared with a conventional patterned ground shield inductor for the implementation of a LC voltage controlled oscillator (VCO) operating at $\mathrm{mmW}$ frequencies. In this work it is shown how the floating-shield inductor achieves higher quality factor and provides a better isolation for substrate-coupled high-frequency interferences.
\end{abstract}

\section{INTRODUCTION}

On-chip inductors are key passive components for CMOS RF and mmW circuits, especially in LC-VCOs where most of their performance is ultimately limited by the quality factor of the inductor used to build the resonant tank. The inductor is usually fabricated using the top metal layers which in CMOS process with RF options are made thicker to reduce the resistance per square. As a consequence, the quality factor is limited by the substrate losses and not by the conductor resistivity. In order to further increase the quality factor and to provide a controlled environment that guarantees a good accuracy of the electrical model, a patterned shield is routinely added under the inductor spirals, made of the lower metal layers and polysilicon [1]. The shield is connected to ground, which guarantees a portable electrical model that is attached to the layout and integrated into a parameterized design kit library.

Recently, the use of floating shields has been proposed as a way to further improve the quality factor of on-chip inductors and transformers [2-4]. Quality factor improvements from 5\% to $15 \%$ are reported, but experimental data available in the literature is limited to frequencies up to $10 \mathrm{GHz}$. In [5] a 60 $\mathrm{GHz}$ VCO using a floating shield under an inductive transmission line is presented. The floating shield is use to change the electromagnetic properties of the structure producing a slow-wave effect which reduces the wavelength and allows the implementation of $\lambda / 4$ shorts (or opens) with reduced dimensions $[2,6]$.

This works was partially funded by EU-Feder and Spanish MICINN TEC2008-01856 project and AGAUR SGR 1497 funds.
In this paper the floating-shield technique is used in a conventional $95 \mathrm{pH}$ spiral inductor by replacing the conventional grounded shield by a floating pattern of M1 and M2 lines under the spiral. This small inductance value is required in circuits operating at $\mathrm{mmW}$ frequencies. Both, the original patterned ground shield and the floating shield inductors have been fabricated and measured up to $\mathrm{mmW}$ frequencies. Next, two versions of a $60 \mathrm{GHz}$ LC-tank VCO are fabricated using each of the inductors and used to investigate the effectiveness of the floating shield in reducing the substrate noise coupling to the VCO in comparison to the patterned ground shield inductor.

\section{INDUCTORS EXPERIMENTAL CHARACTERIZATION}

\section{A. Inductors description}

The inductors analyzed in this work are fabricated in a 1 poly, 6 copper metal layers (the top layer is a thick metal) $65 \mathrm{~nm}$ bulk CMOS process. The inductors are single turn differential inductors of $95 \mathrm{pH}$ DC inductance. The inductor spiral is made with the two upper metal layers plus the aluminum metallization layer and has a conductor width of 5 $\mu \mathrm{m}$, a diameter of $46 \mu \mathrm{m}$ and a total length of $180 \mu \mathrm{m}$. The inductor is provided in the passives library of the commercial design-kit for that process. It comes with a patterned ground shield made of polysilicon and diffusion connected to ground stacked M1 and M2 forming an 'X'-shape (see Fig. 1). This

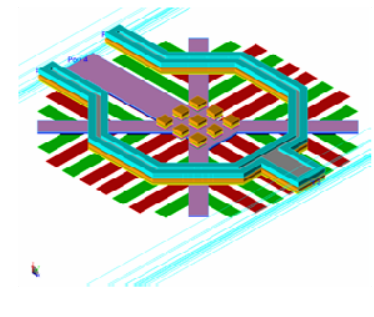

DK Inductor

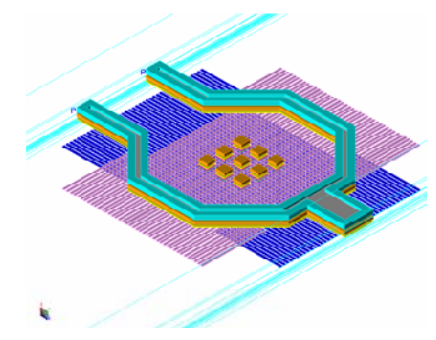

TS Inductor
Figure 1. Patterned ground shield (left) and floating shield (right) inductors $3 \mathrm{D}$ view. 
inductor is labeled 'DK inductor' in the rest of the paper. The inductor is built on top of a P-well where the highconductivity channel stop epi-layer has been suppressed. The floating-shield inductor (labeled 'FS inductor') is obtained after suppressing the patterned ground shield and adding a floating pattern of $0.6 \mu \mathrm{m}$ width metal lines spaced by $0.6 \mu \mathrm{m}$ extending the whole area of the spiral. The pattern is repeated in M1 and M2 layer with orthogonal directions (see Fig. 1 right).

\section{B. Experimental characterization of the inductors}

The two inductors were fabricated in the same chip along with de-embedding standards. Fig. 2 shows the layout and a microphotograph. Measurements were done using a differential mmW $100 \mu \mathrm{m}$ pitch GSGSG probe and a network analyzer. The two-port S-parameters of the two inductors and the de-embedding standards where obtained from $250 \mathrm{MHz}$ to $50 \mathrm{GHz}$. Several calibration and de-embedding procedures were checked. The results shown in this work were obtained from a LRRM calibration at the probe tips followed by an OPEN-SHORT de-embedding [7] using the Short 2 and Open 2 structures shown in Fig. 2. Each set of S-parameters is obtained from the average of four chips found at extreme locations in the same wafer. Finally, the two-port Sparameters are used to obtain one-port differential Sparameters, and then the inductor electrical parameters $(\mathrm{L}$ and $\mathrm{Q}_{\mathrm{s}}$ ), calculated using well known equations.

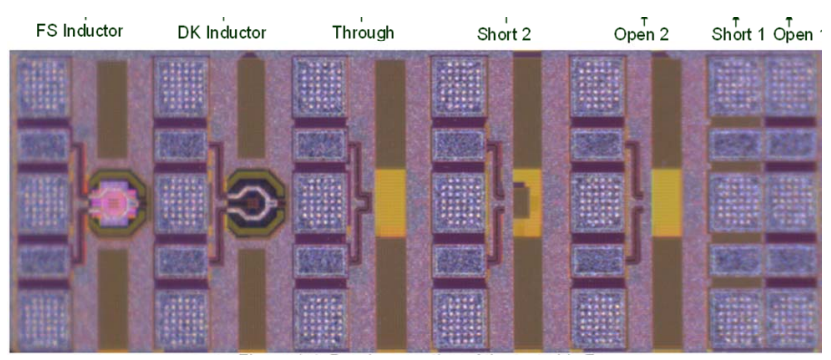

Figure 2. Microphotograph of the inductors characterization chip.

The most important drawback of this type of measurement of such a small components is that the probe pads and access lines limit the frequency range in which the de-embedding process is useful. In the particular case of the inductors used in this work, the access lines required for connecting the differential access port of the inductor to the two signal pads (spaced $200 \mu \mathrm{m}$ ) are $120 \mu \mathrm{m}$ long each and are strongly electromagnetically coupled beyond $25 \mathrm{GHz}$, which avoids having an accurate measurement of the quality factor beyond this frequency. In order to assess the impact of these access structures, the measurements are compared against EM simulations of the inductors (without the pads and access lines) using Agilent Momentum simulator in Fig. 3. The EM simulations have an acceptable match to the quality factor $\left(Q_{s}\right)$ measurements in the region before the self-resonance of the access lines structure, and provide an accurate estimation of the differential inductance in the whole frequency range.
The comparison of the floating shield inductor against the patterned ground shield confirms an important improvement in the quality factor and a slight increase in the inductance $(5$ $\mathrm{pH})$. The $Q_{s}$ of the inductor increases by a factor 1.5 when measured at the access structure resonance $(25 \mathrm{GHz})$. This improvement factor is preserved in the comparison of $Q_{s}$ values at higher frequencies obtained by the EM simulation. This analysis confirms the benefits of using floating shield inductors at $\mathrm{mmW}$ frequencies.
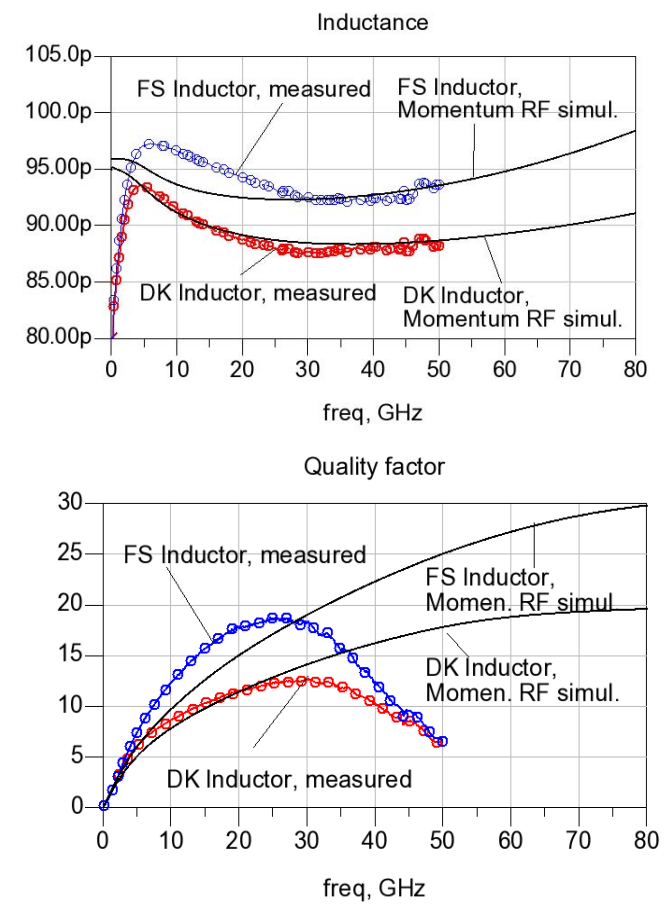

Figure 3. Inductors electrical parameters comparison.

\section{VCO COMPARISON}

Two versions of a $56 \mathrm{GHz}$ LC-Tank VCO were designed and manufactured, one using the DK and the other the FS inductor. The VCO, shown in Fig. 4, is made of an NMOS cross-coupled pair, an LC tank that uses a combination of continuously and binary controlled differential varactors and the differential inductors of the previous section, allowing a $17 \%$ of tuning range [8]. Both versions of the VCO are

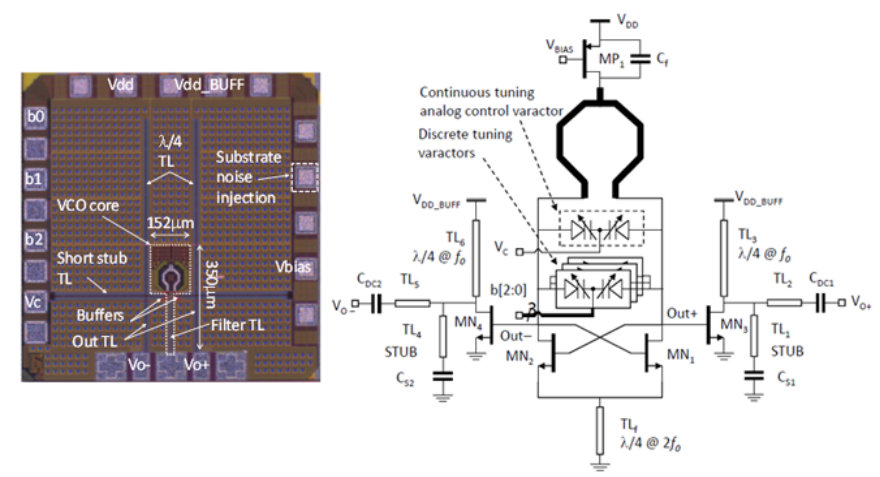

Figure 4. VCO layout and schematic. 
measured in the same conditions consuming $15 \mathrm{~mW}$ from a $1.2 \mathrm{~V}$ supply. Fig. 5 shows the output power of the two oscillators for the whole range of frequencies obtained by the variation of the continuous control voltage and for three digital discrete tuning codes. The oscillation range of the FS inductor $\mathrm{VCO}$ is located at slightly lower frequencies due to the increase of the tank inductance, and its output power is around $2 \mathrm{~dB}$ higher, confirming the increase of the quality factor.
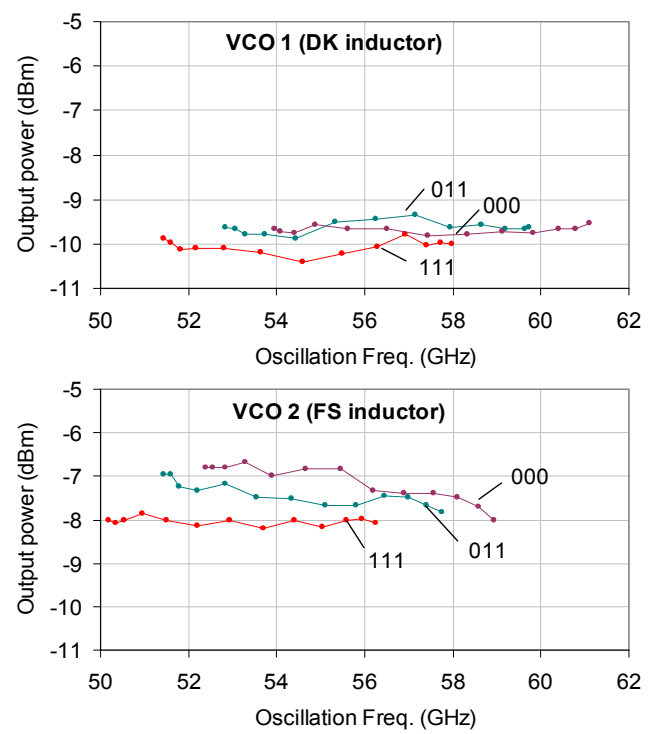

Figure 5. Impact on VCO output power of the DK and FS inductors.

\section{SUBSTRATE NOISE ISOLATION COMPARISON}

The patterned ground shield has been proposed as an efficient way of reducing the substrate noise impact on VCOs by reducing the coupling of such noise to the inductor $[9,10]$. The effectiveness of this technique relies, however, in the biasing impedance used to connect the shield to a clean ground. For high frequency substrate noise, for example the one generated by another VCO or the PA present in the same chip, the ground shield of the inductors is not very efficient due to the inductive nature of the chip to board ground connections. A floating shield offers a better isolation, as is shown in the following experimental results.

The layout of the two VCOs presented in the previous section has an extra pad to contact the substrate using a large array of $\mathrm{p}+$ diffusion contacts. This pad is connected to a $\mathrm{mmW}$ sinusoidal generator and used to inject a pure tone into the substrate at a frequency close, but not equal, to the oscillation frequency of the VCO. It has been already shown that this type of substrate interference produces two sidebands at an offset frequency equal to $\pm\left(f_{n}-f_{o}\right)$ where $f_{o}$ is the VCO oscillation frequency and $f_{n}$ is the single tone injected into the substrate [11]. Fig. 6 shows an example of the output spectrum of the DK inductor VCO perturbed by high frequency substrate noise of $50.195 \mathrm{GHz}$ when it is oscillating at $50.170 \mathrm{GHz}$ (power levels are not corrected with the probe and cable losses). The upper sideband falls at the same frequency as the injected tone. The VCO frequency $f_{o}$ drifts during the the spectrum analyzer averaging process because of the high sensitivity of the VCO to power supply noise $(10 \mathrm{GHz} / \mathrm{V})$, which is a consequence of its large continuous tuning range. Such a drift produces a widening of the VCO output spectrum that is doubled in the lower sideband. Although apparently the sideband spurs show different peak values, their power levels are always the same when integrated over the sideband area.

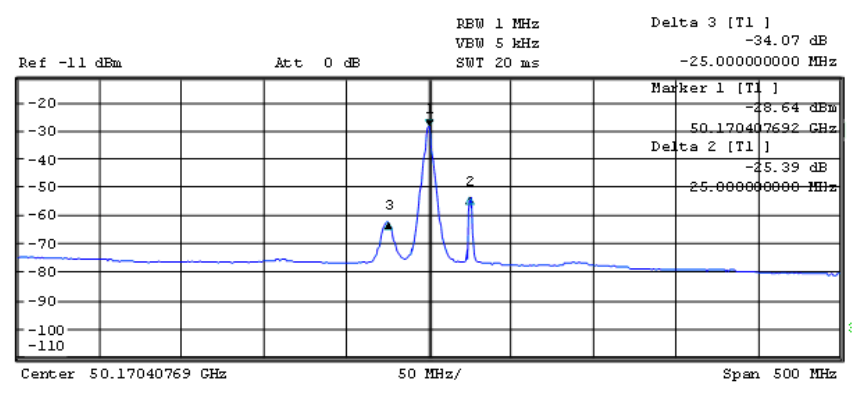

Figure 6. VCO sideband spurs due high-frequency substrate noise.

In the following experiment the injected substrate tone $f_{n}$ is varied so that the spurs appear at offsets from $f_{o}$ ranging from $10 \mathrm{MHz}$ to $150 \mathrm{MHz}$, keeping constant the injected tone power. The same measurements are repeated for both the DK and the FS inductor VCOs. Fig. 7 compares the spurs power relative to the VCO output power in the two VCOs. The figure shows the relative upper sideband (USB) spur amplitude measured at different control voltages (i.e. for different oscillation frequencies). It has a $-20 \mathrm{~dB} / \mathrm{dec}$ dependence on offset frequency indicating that the spurs are an FM modulation of the VCO output. Moreover, they depend weakly on the VCO control voltage $\left(V_{c}\right)$, and therefore they can not be due to direct coupling to this signal. Indeed, the VCO incorporates on-chip capacitors between all the DC inputs $\left(V_{D D}\right.$, current bias control and VCO frequency control voltages) and ground. At the frequencies of the injected substrate noise such capacitors guarantee than no differential voltage appears between these nodes that could produce FM modulation. As a consequence, the inductor is the dominant component that couples the substrate noise into the VCO. The comparison between the two VCOs indicates that the spurs amplitude is reduced by $6 \mathrm{dBs}$ in the case of the floating shield in comparison to the patterned ground shield, thus confirming another important benefit of the floating shield inductor in front of the conventional patterned ground shield inductor. 

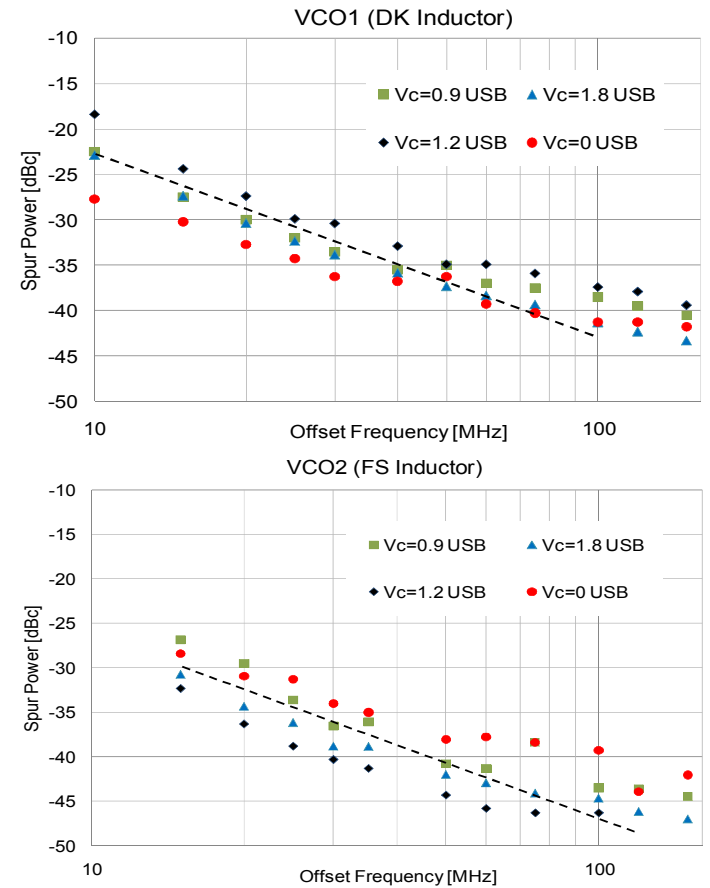

Figure 7. Comparison of sideband spurs amplitude due to high-frequency substrate noise coupled to the VCO through the inductors.

\section{CONCLUSION}

In this work it has been shown experimentally that floating shield inductors have two major benefits in comparison to the conventional patterned ground shield inductors for the implementation of mmW VCOs. Firstly, the inductor quality factor is observed to increase by a factor of 1.5. The result obtained by the measurement and EM simulation of the inductors is confirmed by the characterization of a VCO containing the same inductor with both types of shield. This is the first time, to best of the knowledge of the authors, that such a large increase is observed for spiral inductors at $\mathrm{mmW}$ frequencies. Secondly, the floating shield demonstrates a better isolation strategy for reducing the coupling to the VCO of high-frequency components of substrate noise. Although the measurements are restricted to a $\mathrm{mmW} \mathrm{VCO}$, the conclusions of this study can be extrapolated to other circuits operating at $\mathrm{mmW}$ frequencies requiring the use of spiral inductors, such as LNAs, Mixers, PAs, transformers or filters.

\section{ACKNOWLEDGEMENT}

The authors would like to tank Nathalie Rolland and Elisabeth DELOS from IEMN, Villeneuve d'Ascq, France, for support on the measurements.

\section{REFERENCES}

[1] C. Patrick Yue, S. Simon Wong, "On-Chip Spiral Inductors with Patterned Ground Shields for Si-Based RF IC's," IEEE J. of Solid-State Circuits, vol. 33, No. 5, May 1998, pp. 743-752.

[2] Tak Shun Dickson, John R. Long, "Shielded Passive Devices for Silicon-Based Monolitc Microwave and Millimeter-Wave Integrated Circcuits," IEEE J. of Solid-State Circuits, vol. 41, No. 5, May 2006, pp. 1183-1194.

[3] C.B. Sia, et al, "Effets of Polysilicon Shield on Spiral Inductors for Silicon-Based RF IC's," Proc. of Intl. Symp. on VLSI Technology, Systems, and Applications, pp. 158-181, April 2001.

[4] Ronal L. Haner, Shoba Kishnan, Sean T. Burns, "Spiral Inductors With Projected Floating Shields: An Alternative Method for RF Shielding," Proc. of IEEE Intl. Symp. on Circuits and Systems, pp. 1771-1774, May 2009.

[5] J. Boremans, et al, "VCO design for $60 \mathrm{GHz}$ applications using differential shielded inductors in 0.13um CMOS," IEEE RFIC Symp. Dig., pp. 125-138, June 2008.

[6] Ivan C.H. Lai, Minoru Fujishima, "High-Q Slow-Wave Transmission Line for Chip Area Reduction on Advanced CMOS Processes," IEEE Intl. Conf. on Microelectronic Test Structures, pp. 19.22, March 2007.

[7] M.C.A.M. Koolen, J.A.M. Geelen, M.P.J.G. Versleijen, “An Improved De-Embedding Technique for On-Wafer Hihg-Frequency Characterization," Proc. of Bipolar Circuits and Technology Meeting, pp. 188-191, Sept. 1991.

[8] J.L. González, F. Badets, B. Martineau, D. Belot, "A 56GHz LC-Tank VCO with $17 \%$ Tuning Range in 65nm Bulk CMOS for Wireless HDMI Applications," IEEE RFIC Symp. Dig., pp. 481-484, June 2009.

[9] H. Shen, J. Wen. X. Duo, X. Lv, "Substrate Noise Suppresion by PGS of Inductors," Proc. of Asia-Pacific Microwave Conference, 2007.

[10] Y.C. Wu, S.S.H: Hsu, K. K.W. Tan, Y.S. Su, "Substrate Noise Coupling Reduction in LC Voltage-Controlled Oscillators," IEEE Electron Device Letters, vol. 30, no. 4, April 2009, pp. 383-385.

[11] M.A. Méndez, D. Mateo, X. Aragonés, J.L. González, "Phase noise degradation of LC-tank VCOs due to substrate noise and package coupling," Proc. IEEE European Solid-State Conference, pp. 105-108, 2005 . 